\title{
MIXTURE REPRESENTATIONS OF RESIDUAL LIFETIMES OF USED SYSTEMS
}

\author{
JORGE NAVARRO, ${ }^{*}$ Universidad de Murcia \\ N. BALAKRISHNAN, ${ }^{* *}$ McMaster University \\ FRANCISCO J. SAMANIEGO, ${ }^{* * *}$ University of California, Davis
}

\begin{abstract}
The representation of the reliability function of the lifetime of a coherent system as a mixture of the reliability function of order statistics associated with the lifetimes of its components is a very useful tool to study the ordering and the limiting behaviour of coherent systems. In this paper, we obtain several representations of the reliability functions of residual lifetimes of used coherent systems under two particular conditions on the status of the components or the system in terms of the reliability functions of residual lifetimes of order statistics.
\end{abstract}

Keywords: Coherent system; $k$-out-of- $n$ system; order statistics; signature; stochastic ordering

2000 Mathematics Subject Classification: Primary 60E15

Secondary $60 \mathrm{~K} 10$

\section{Introduction}

A coherent system is one in which every component is relevant (that is, actually affects the working or failure of the system) and for which the structure function is monotone in every component (that is, replacing a failed component by a working component cannot cause a working system to fail). Although there has been some work in reliability that focuses on monotone systems, most work has been restricted to the class of coherent systems. This restriction is motivated by the reasoning that if the relevance or monotonicity restrictions are violated for a given system, we could design a coherent system with the same number of components (or fewer) that achieves the same or better performance.

The signature of a coherent system is a very useful tool to study the system behaviour or to compare the behaviour of different systems. The utility of signatures is evident from the following mixture representation theorem due to Samaniego [12] (see also [13, p. 25]). It shows that the lifetime distribution of a coherent system based on $n$ components with independent and identically distributed (i.i.d.) lifetimes and common continuous distribution $F$ can be written as a function which depends on the system's design only through its signature.

Theorem 1.1. Consider a coherent system based on $n$ components with i.i.d. lifetimes $X_{1}, X_{2}$, $\ldots, X_{n}$ distributed according to the common continuous distribution $F$. Let $T$ be the system's

\footnotetext{
Received 23 May 2008; revision received 22 September 2008.

* Postal address: Facultad de Matemáticas, Universidad de Murcia, 30100 Murcia, Spain.

Email address: jorgenav@um.es

** Postal address: Department of Mathematics and Statistics, McMaster University, Hamilton, Ontario L8S 4K1,

Canada. Email address: bala@mcmaster.ca

*** Postal address: University of California, Davis, 1 Shields Avenue, Davis, CA 95616, USA.

Email address: fjsamaniego@ucdavis.edu
} 
lifetime. Then

$$
\bar{F}_{T}(t)=\sum_{i=1}^{n} s_{i} \mathrm{P}\left(X_{i: n}>t\right),
$$

where $\bar{F}_{T}(t)=\mathrm{P}(T>t), X_{1: n}, X_{2: n}, \ldots, X_{n: n}$ are the order statistics obtained from $X_{1}, X_{2}$, $\ldots, X_{n}, s_{i}=\mathrm{P}\left(T=X_{i: n}\right)$, and $s=\left(s_{1}, \ldots, s_{n}\right)$ is the system's signature.

Mixture representations have proven especially useful in the comparison of the performance of competing systems. For example, Kochar et al. [5] established the following preservation results. Here, ' $\leq_{\mathrm{st}}$ ', ' $\leq_{\mathrm{hr}}$ ' and ' $\leq_{\mathrm{lr}}$ ' refer to the stochastic, hazard rate, and likelihood ratio orderings, respectively; for details on these orderings, we refer the reader to Chapter 1 of [15].

Theorem 1.2. Let $s_{1}$ and $s_{2}$ be the signatures of two coherent systems, both based on $n$ components with i.i.d. lifetimes distributed according to the common continuous distribution $F$. Let $T_{1}$ and $T_{2}$ be their respective lifetimes.

(a) If $s_{1} \leq_{\text {st }} s_{2}$ then $T_{1} \leq_{\text {st }} T_{2}$.

(b) If $s_{1} \leq_{\mathrm{hr}} \boldsymbol{s}_{2}$ then $T_{1} \leq_{\mathrm{hr}} T_{2}$.

(c) If $F$ is absolutely continuous and $s_{1} \leq_{\operatorname{lr}} s_{2}$, then $T_{1} \leq_{\operatorname{lr}} T_{2}$.

These results can be extended to the concept of mixed systems, that is, to stochastic mixtures of coherent systems; see [2] for more details. Hence, a mixture representation similar to the one in (1.1) holds for the reliability function of the lifetime of any mixed system and Theorem 1.2 applies to these kinds of systems as well.

The purpose of this paper is to extend these results to the conditional distributions of systems under certain conditions on the components or the system state.

The paper is organised as follows. In Section 2, we present some mixture representations for the residual lifetime $(T-t \mid T>t)$ of a system with i.i.d. component lifetimes. In Section 3 we do the same under additional conditions on the components. Specifically, we consider the residual lifetime $\left(T-t \mid T>t, X_{i: n}<t\right)$, a situation in which we know that at time $t$ the system is working but at least $i$ components have failed. Finally, in Section 4 we provide some conclusions and some problems that are worth considering for further investigation.

Throughout this paper, the notions 'increasing' and 'decreasing' are used in the weak sense (i.e. a function $g$ is increasing if $g(x) \leq g(y)$ for all $x<y$ ).

\section{Mixture representations of residual lifetimes of used systems}

Let $T=\phi\left(X_{1}, X_{2}, \ldots, X_{n}\right)$ be the lifetime of a coherent system with i.i.d. component lifetimes $X_{1}, X_{2}, \ldots, X_{n}$ from a common continuous distribution function $F$. Let $\bar{F}=1-F$ be the common reliability function, and let $X_{1: n}, X_{2: n}, \ldots, X_{n: n}$ be the corresponding order statistics. In this section we establish representations similar to that in (1.1) for the reliability function of a used but working system, i.e. for the distribution of the system lifetime $T$ given that it is known that $T>t$ or, equivalently, for the residual lifetime $(T-t \mid T>t)$. From (1.1) we have

$$
\mathrm{P}(T-t>x \mid T>t)=\frac{\bar{F}_{T}(t+x)}{\bar{F}_{T}(t)}=\frac{\sum_{i=1}^{n} s_{i} \mathrm{P}\left(X_{i: n}>t+x\right)}{\sum_{i=1}^{n} s_{i} \mathrm{P}\left(X_{i: n}>t\right)}
$$

for all $x \geq 0$ and $t \geq 0$ such that $\bar{F}_{T}(t)>0$. Since

$$
\mathrm{P}\left(X_{i: n}>t+x \mid X_{i: n}>t\right) \mathrm{P}\left(X_{i: n}>t\right)=\mathrm{P}\left(X_{i: n}>t+x\right),
$$


this expression can also be written as

$$
\mathrm{P}(T-t>x \mid T>t)=\sum_{i=1}^{n} p_{i}(t) \mathrm{P}\left(X_{i: n}-t>x \mid X_{i: n}>t\right)
$$

where $p_{i}(t)=s_{i} \mathrm{P}\left(X_{i: n}>t\right) / \bar{F}_{T}(t)$. The function $p_{i}(t)$ may be identified as the probability of $\left\{T=X_{i: n} \mid T>t\right\}$ as follows:

$$
\begin{aligned}
\mathrm{P}\left(T=X_{i: n} \mid T>t\right) & =\frac{\mathrm{P}\left(T>t, T=X_{i: n}\right)}{\bar{F}_{T}(t)} \\
& =\frac{\mathrm{P}\left(T=X_{i: n}\right) \mathrm{P}\left(T>t \mid T=X_{i: n}\right)}{\bar{F}_{T}(t)} \\
& =\frac{\mathrm{P}\left(T=X_{i: n}\right) \mathrm{P}\left(X_{i: n}>t \mid T=X_{i: n}\right)}{\bar{F}_{T}(t)} \\
& =\frac{s_{i} \mathrm{P}\left(X_{i: n}>t\right)}{\bar{F}_{T}(t)} \\
& =p_{i}(t),
\end{aligned}
$$

where $\mathrm{P}\left(X_{i: n}>t \mid T=X_{i: n}\right)=\mathrm{P}\left(X_{i: n}>t\right)$ follows from the fact that the events $\left\{T=X_{i: n}\right\}$ and $X_{i: n}>t$ are independent when the component lifetimes are i.i.d.

This reveals that the residual lifetime $(T-t \mid T>t)$ of the system at time $t$ is a mixture of the residual lifetimes $\left(X_{i: n}-t \mid X_{i: n}>t\right)$ of the order statistics (which represent the lifetimes of $k$-out-of- $n$ systems) at time $t$ with coefficients $p_{i}(t)$ for $i=1,2, \ldots, n$. Note that the vector $\boldsymbol{p}(t)=\left(p_{1}(t), p_{2}(t), \ldots, p_{n}(t)\right)$ of coefficients is the conditional distribution of the index $I_{t}$ of the ordered component lifetime that is fatal to the system given that $T>t$. These coefficients depend both on $\phi$ and on $F(t)$. Furthermore, we also note that $(T-t \mid T>t)$ is equal in distribution to a mixed system of the residual lifetimes of $k$-out-of- $n$ systems. For example, if we consider the system with lifetime $T=\min \left(X_{1}, \max \left(X_{2}, X_{3}\right)\right)$ and corresponding signature $s=\left(\frac{1}{3}, \frac{2}{3}, 0\right)$, and that we know that it is working at time $t$, then

$$
\boldsymbol{p}(t)=\left(\frac{\bar{F}(t)}{6-3 \bar{F}(t)}, \frac{6-4 \bar{F}(t)}{6-3 \bar{F}(t)}, 0\right) .
$$

Note that $\boldsymbol{p}(0)=\boldsymbol{s}=\left(\frac{1}{3}, \frac{2}{3}, 0\right)$ and that $\lim _{t \rightarrow \infty} \boldsymbol{p}(t)=(0,1,0)$, that is, the residual lifetime of the system $(T-t \mid T>t)$ is asymptotically (as $t \rightarrow \infty)$ equivalent to the residual lifetime $\left(X_{2: 3}-t \mid X_{2: 3}>t\right)$ of the 2-out-of-3 system (i.e. they have the same law as $\left.t \rightarrow \infty\right)$; see [7], [8], and [11] for analogous results based on the hazard rate and mean residual life functions.

Under the i.i.d. assumption on component lifetimes, the representation in (2.1) can be extended to mixed systems and, in particular, to coherent systems with fewer than $n$ components since they are equal in distribution to mixed systems with $n$ components; see [10] or Theorem 3.2 of [13]. Thus, for example, the series system with two components $X_{1: 2}$ is equal in distribution to the mixed system with signature $s_{1: 2}=\left(\frac{2}{3}, \frac{1}{3}, 0\right)$, and, hence, $(2.1)$ holds with

$$
p_{1: 2}(t)=\left(\frac{2 \bar{F}(t)}{3}, 1-\frac{2 \bar{F}(t)}{3}, 0\right) .
$$


TABLE 1: Vectors of coefficients in (2.1) with $n=3$ for coherent systems with 1-3 i.i.d. components.

\begin{tabular}{|c|c|c|}
\hline System & $T=\phi\left(X_{1}, X_{2}, X_{3}\right)$ & $\boldsymbol{p}(t)$ \\
\hline 1 & $X_{1: 1}=X_{1}$ & $\left(\frac{1}{3} \bar{F}^{2}(t), \bar{F}(t)-\frac{2}{3} \bar{F}^{2}(t), 1-\bar{F}(t)+\frac{1}{3} \bar{F}^{2}(t)\right)$ \\
\hline 2 & $X_{1: 2}=\min \left(X_{1}, X_{2}\right)$ & $\left(\frac{2}{3} \bar{F}(t), 1-\frac{2}{3} \bar{F}(t), 0\right)$ \\
\hline 3 & $X_{2: 2}=\max \left(X_{1}, X_{2}\right)$ & $\left(0, \frac{3 \bar{F}(t)-2 \bar{F}^{2}(t)}{6-3 \bar{F}(t)}, \frac{6-6 \bar{F}(t)+2 \bar{F}^{2}(t)}{6-3 \bar{F}(t)}\right)$ \\
\hline 4 & $X_{1: 3}=\min \left(X_{1}, X_{2}, X_{3}\right)$ & $(1,0,0)$ \\
\hline 5 & $\min \left(X_{1}, \max \left(X_{2}, X_{3}\right)\right)$ & $\left(\frac{\bar{F}(t)}{6-3 \bar{F}(t)}, \frac{6-4 \bar{F}(t)}{6-3 \bar{F}(t)}, 0\right)$ \\
\hline 6 & $X_{2: 3}$ (2-out-of-3) & $(0,1,0)$ \\
\hline 7 & $\max \left(X_{1}, \min \left(X_{2}, X_{3}\right)\right)$ & $\left(0, \frac{6 \bar{F}(t)-4 \bar{F}^{2}(t)}{3+3 \bar{F}(t)-3 \bar{F}^{2}(t)}, \frac{3-3 \bar{F}(t)+\bar{F}^{2}(t)}{3+3 \bar{F}(t)-3 \bar{F}^{2}(t)}\right)$ \\
\hline 8 & $X_{3: 3}=\max \left(X_{1}, X_{2}, X_{3}\right)$ & $(0,0,1)$ \\
\hline
\end{tabular}

In Table 1, we have presented the vectors of coefficients of order 3 in (2.1) for coherent systems with 1-3 i.i.d. components. It is well known that if a system $T$ has signature $\left(s_{1}, s_{2}, \ldots, s_{n}\right)$ then the signature of the dual system is $\left(s_{n}, s_{n-1}, \ldots, s_{1}\right)$; see [5]. However, we observe from Table 1 that this property is not necessarily true for the vectors of coefficients in (2.1), as illustrated by systems 5 and 7 . We can obtain a result similar to Theorem 1.2 for mixed systems and representation (2.1) as follows.

Theorem 2.1. Let $\boldsymbol{p}_{1}(t)$ and $\boldsymbol{p}_{2}(t)$ be the vectors of coefficients in representation (2.1), for a fixed $t \geq 0$, of two mixed systems, both based on $n$ components with i.i.d. lifetimes distributed according to the common continuous distribution $F$. Let $T_{1}$ and $T_{2}$ be their respective lifetimes.

(a) If $\boldsymbol{p}_{1}(t) \leq_{\mathrm{st}} \boldsymbol{p}_{2}(t)$ then $\left(T_{1}-t \mid T_{1}>t\right) \leq_{\mathrm{st}}\left(T_{2}-t \mid T_{2}>t\right)$.

(b) If $\boldsymbol{p}_{1}(t) \leq_{\mathrm{hr}} \boldsymbol{p}_{2}(t)$ then $\left(T_{1}-t \mid T_{1}>t\right) \leq_{\mathrm{hr}}\left(T_{2}-t \mid T_{2}>t\right)$.

(c) If $F$ is absolutely continuous and $p_{1}(t) \leq \operatorname{lr} p_{2}(t)$, then $\left(T_{1}-t \mid T_{1}>t\right) \leq_{\operatorname{lr}}\left(T_{2}-\right.$ $\left.t \mid T_{2}>t\right)$.

Proof. It is well known that the order statistics are likelihood ratio ordered in the i.i.d. case, i.e. $X_{i: n} \leq_{\operatorname{lr}} X_{i+1: n}$ for $i=1,2, \ldots, n-1$ (see [6] or [15, p. 54]). Moreover, it is also known that if two random variables are lr-ordered, so are their residual lifetimes; see, for example, Theorem 1.C.6 of [15]. Hence, we have

$$
\left(X_{i: n}-t \mid X_{i: n}>t\right) \leq_{\operatorname{lr}}\left(X_{i+1: n}-t \mid X_{i+1: n}>t\right) \quad \text { for all } i=1,2, \ldots, n-1 .
$$

Therefore, these residual lifetimes are also hr- and st-ordered. The proof is completed by using (2.1) and following the steps of Theorem 1.2 (see [5]).

For example, note that

$$
\left(\frac{2}{3} \bar{F}(t), 1-\frac{2}{3} \bar{F}(t), 0\right) \leq \operatorname{lr}\left(\frac{\bar{F}(t)}{6-3 \bar{F}(t)}, \frac{6-4 \bar{F}(t)}{6-3 \bar{F}(t)}, 0\right)
$$


for arbitrary $t \geq 0$ and, hence, from Table 1 and Theorem 2.1, if $F$ is absolutely continuous, we have

$$
\left(X_{1: 2}-t \mid X_{1: 2}>t\right) \leq_{\mathrm{lr}}(T-t \mid T>t) \quad \text { for all } t \geq 0,
$$

where $T=\min \left(X_{1}, \max \left(X_{2}, X_{3}\right)\right)$. Note, however, that they are equivalent when $t \rightarrow \infty$. The next example shows that Theorem 2.1 can also be used to determine the age $t$ at which a used system is st-better than yet another used system when the two are not ordered for all $t$.

Example 2.1. Let us consider the systems $X_{1: 2}$ and $T=\min \left(X_{2: 3}, X_{4}\right)$ with corresponding signatures of order 4 as $s_{1: 2}=\left(\frac{1}{2}, \frac{1}{3}, \frac{1}{6}, 0\right)$ and $s=\left(\frac{1}{4}, \frac{3}{4}, 0,0\right)$ (see Table 1 of [10]). It is then easy to see that $X_{1: 2}$ and $T$ are not st-ordered. Their respective vectors of coefficients in (2.1) can be found to be

$$
\boldsymbol{p}_{1: 2}(t)=\left(\frac{\bar{F}^{2}(t)}{2}, \frac{4 \bar{F}(t)-3 \bar{F}^{2}(t)}{3}, 1-\frac{8 \bar{F}(t)-3 \bar{F}^{2}(t)}{6}, 0\right)
$$

and

$$
\boldsymbol{p}(t)=\left(\frac{\bar{F}(t)}{12-8 \bar{F}(t)}, \frac{12-9 \bar{F}(t)}{12-8 \bar{F}(t)}, 0,0\right) .
$$

It is easy to verify that $\boldsymbol{p}_{1: 2}(t) \geq_{\text {st }} \boldsymbol{p}(t)$ whenever

$$
\bar{F}(t) \leq \frac{3-\sqrt{5}}{4} .
$$

Consequently, if $t_{1}=\inf \{t \geq 0: \bar{F}(t)=(3-\sqrt{5}) / 4\}$ then $\boldsymbol{p}_{1: 2}(t) \geq$ st $\boldsymbol{p}(t)$ for all $t \geq t_{1}$. Hence, from Theorem 2.1 we have $\left(X_{1: 2}-t \mid X_{1: 2}-t\right) \geq_{\text {st }}(T-t \mid T>t)$ for all $t \geq t_{1}$. Note that they are not st-ordered for $0 \leq t<t_{1}$ and that

$$
\lim _{t \rightarrow \infty} \boldsymbol{p}_{1: 2}(t)=(0,0,1,0) \geq_{\mathrm{st}} \lim _{t \rightarrow \infty} \boldsymbol{p}(t)=(0,1,0,0) .
$$

The next result proves that any coherent system has its tail stochastic behaviour similar to that of an order statistic (i.e. of a $k$-out-of- $n$ system). Hence, in the limit as $t \rightarrow \infty$, the st-order is a total order in the set of the residual lifetimes of mixed systems.

Theorem 2.2. If $T$ is a coherent system with signature $s=\left(s_{1}, s_{2}, \ldots, s_{i}, 0,0, \ldots, 0\right)$, where $s_{i}>0$ for an integer $i \in\{1,2, \ldots, n\}$, then

$$
\lim _{t \rightarrow \infty} p(t)=(\underbrace{0,0, \ldots, 0}_{i-1 \text { times }}, 1, \underbrace{0,0, \ldots, 0}_{n-i \text { times }}) .
$$

Proof. From (1.1), we have

$$
\lim _{t \rightarrow \infty} p_{k}(t)=s_{k} \lim _{t \rightarrow \infty} \frac{\mathrm{P}\left(X_{k: n}>t\right)}{\bar{F}_{T}(t)}=s_{k} \lim _{t \rightarrow \infty} \frac{\mathrm{P}\left(X_{k: n}>t\right)}{\sum_{r=1}^{i} s_{r} \mathrm{P}\left(X_{r: n}>t\right)} .
$$

Since (see [3, p. 46])

$$
\mathrm{P}\left(X_{r: n}>t\right)=\sum_{j=n-r+1}^{n}(-1)^{j-n+r-1}\left(\begin{array}{l}
j-1 \\
n-r
\end{array}\right)\left(\begin{array}{l}
n \\
j
\end{array}\right) \bar{F}^{j}(t),
$$


then

$$
\lim _{t \rightarrow \infty} \frac{\mathrm{P}\left(X_{r: n}>t\right)}{\mathrm{P}\left(X_{k: n}>t\right)}= \begin{cases}0 & \text { if } r<k \\ 1 & \text { if } r=k \\ +\infty & \text { if } r>k\end{cases}
$$

So

$$
\lim _{t \rightarrow \infty} p_{k}(t)=s_{k} \lim _{t \rightarrow \infty} \frac{\mathrm{P}\left(X_{k: n}>t\right)}{\sum_{r=1}^{i} s_{r} \mathrm{P}\left(X_{r: n}>t\right)}= \begin{cases}0 & \text { if } k \neq i, \\ 1 & \text { if } k=i,\end{cases}
$$

which proves the result.

We shall now establish some extensions of Theorem 2.1. The first one will enable us to compare the residual lifetimes of used systems with different components.

Theorem 2.3. Let $\boldsymbol{p}_{1}(t)$ and $\boldsymbol{p}_{2}(t)$ be the vectors of coefficients in (2.1), for a fixed $t \geq 0$, of two mixed systems with $n$ i.i.d. component lifetimes $X_{1}, \ldots, X_{n}$ and $Y_{1}, \ldots, Y_{n}$, distributed according to common continuous distributions $F$ and $G$, respectively. Let $T_{1}$ and $T_{2}$ be their respective lifetimes.

(a) If $F \leq_{\mathrm{hr}} G$ and $\boldsymbol{p}_{1}(t) \leq_{\mathrm{st}} \boldsymbol{p}_{2}(t)$, then $\left(T_{1}-t \mid T_{1}>t\right) \leq_{\mathrm{st}}\left(T_{2}-t \mid T_{2}>t\right)$.

(b) If $F \leq \mathrm{hr} G$ and $p_{1}(t) \leq_{\mathrm{hr}} p_{2}(t)$, then $\left(T_{1}-t \mid T_{1}>t\right) \leq_{\mathrm{hr}}\left(T_{2}-t \mid T_{2}>t\right)$.

(c) If $F$ and $G$ are absolutely continuous, $F \leq_{\operatorname{lr}} G$, and $p_{1}(t) \leq_{\operatorname{lr}} p_{2}(t)$, then $\left(T_{1}-t \mid T_{1}>\right.$ $t) \leq \operatorname{lr}\left(T_{2}-t \mid T_{2}>t\right)$.

Proof. If $F \leq{ }_{\mathrm{hr}} G$ then $X_{i: n} \leq \mathrm{hr} Y_{i: n}$ and so

$$
\left(X_{i: n}-t \mid X_{i: n}>t\right) \leq_{\mathrm{hr}}\left(Y_{i: n}-t \mid Y_{i: n}>t\right) \quad \text { for all } i=1,2, \ldots, n
$$

see $[15$, p. 34]. Hence, they are also st-ordered. Then, (a) and (b) are obtained from Theorems 1.A.6 and 1.B.14 of [15], respectively.

If $F \leq \operatorname{lr} G$ then $X_{i: n} \leq_{\operatorname{lr}} Y_{i: n}$ and so

$$
\left(X_{i: n}-t \mid X_{i: n}>t\right) \leq \operatorname{lr}\left(Y_{i: n}-t \mid Y_{i: n}>t\right) \text { for all } i=1,2, \ldots, n \text {; }
$$

see [15, p. 55]. Then, (c) is obtained from Theorem 1.C.17 of [15]. This completes the proof.

The following theorem allows us to compare the residual lifetimes of a system at different ages. But, we first need a lemma which gives an intuitive property that the conditional distribution of $I_{t}$ determined by the vector of coefficients $\boldsymbol{p}(t)$ in (2.1) is stochastically increasing in $t$, that is, $I_{t_{1}} \leq_{\text {st }} I_{t_{2}}$ for all $t_{1} \leq t_{2}$. In particular, as $\boldsymbol{s}=\boldsymbol{p}(0)$, it also shows that $\boldsymbol{s} \leq_{\mathrm{st}} \boldsymbol{p}(t)$.

Lemma 2.1. Let $\boldsymbol{p}(t)$ be the vector of coefficients in (2.1) of a mixed system with $n$ i.i.d. components. Then, $\boldsymbol{p}\left(t_{1}\right) \leq \mathrm{st} \boldsymbol{p}\left(t_{2}\right)$ for all $0 \leq t_{1} \leq t_{2}$.

Proof. We need to show that

$$
\sum_{k=j}^{n} \frac{s_{k} \bar{F}_{k: n}\left(t_{1}\right)}{\bar{F}_{T}\left(t_{1}\right)} \leq \sum_{k=j}^{n} \frac{s_{k} \bar{F}_{k: n}\left(t_{2}\right)}{\bar{F}_{T}\left(t_{2}\right)}
$$


for all $j=2,3, \ldots, n$ and all $0 \leq t_{1} \leq t_{2}$. Using (1.1), straightforward algebra shows that (2.2) is equivalent to

$$
\sum_{i=1}^{j-1} \sum_{k=j}^{n} s_{i} s_{k}\left\{\bar{F}_{i: n}\left(t_{1}\right) \bar{F}_{k: n}\left(t_{2}\right)-\bar{F}_{k: n}\left(t_{1}\right) \bar{F}_{i: n}\left(t_{2}\right)\right\} \geq 0,
$$

since

$$
\sum_{i=j}^{n} \sum_{k=j}^{n} s_{i} s_{k}\left\{\bar{F}_{i: n}\left(t_{1}\right) \bar{F}_{k: n}\left(t_{2}\right)-\bar{F}_{k: n}\left(t_{1}\right) \bar{F}_{i: n}\left(t_{2}\right)\right\}=0 .
$$

Finally, using the fact that the order statistics from i.i.d. random variables are hr-ordered, that is, $X_{i: n} \leq_{\mathrm{hr}} X_{k: n}$ for $i \leq k$, we have $\bar{F}_{i: n}(t) / \bar{F}_{k: n}(t)$ to be decreasing in $t$ (see [15, p. 16]), and then

$$
\bar{F}_{i: n}\left(t_{1}\right) \bar{F}_{k: n}\left(t_{2}\right)-\bar{F}_{k: n}\left(t_{1}\right) \bar{F}_{i: n}\left(t_{2}\right) \geq 0
$$

for all $t_{1} \leq t_{2}$ and $i \leq k$. This completes the proof.

Theorem 2.4. Let $\boldsymbol{p}(t)$ be the vector of coefficients in (2.1) of a mixed system based on $n$ components with i.i.d. lifetimes $X_{1}, X_{2}, \ldots, X_{n}$, and let $T$ be the lifetime of the system.

(a) If $X_{i: n} \leq_{\mathrm{st}}\left(X_{i: n}-t \mid X_{i: n}>t\right)$ for a fixed $t>0$ and all $i=1,2, \ldots, n$, then $T \leq_{\mathrm{st}}(T-t \mid T>t)$.

(b) If $\left(X_{i: n}-t_{1} \mid X_{i: n}>t_{1}\right) \leq_{\mathrm{st}}\left(X_{i: n}-t_{2} \mid X_{i: n}>t_{2}\right)$ for fixed $t_{1}<t_{2}$ and all $i=$ $1,2, \ldots, n$, then $\left(T-t_{1} \mid T>t_{1}\right) \leq_{\text {st }}\left(T-t_{2} \mid T>t_{2}\right)$.

The proof is immediate from (2.1), Lemma 2.1 presented above, and Theorem 1.A.6 of [15, p. 7]. Note that (a) shows that if $X_{i: n} \leq_{\text {st }}\left(X_{i: n}-t \mid X_{i: n}>t\right)$ for all $t \in\left(0, t_{1}\right)$ and all $i=1,2, \ldots, n$, then $T \leq_{\text {st }}(T-t \mid T>t)$ for all $t \in\left(0, t_{1}\right)$. This property can be used to obtain a burn-in period for system $T$, i.e. to obtain the maximum value $t_{\mathrm{m}}$ such that $T \leq_{\mathrm{st}}(T-t \mid T>t)$ holds for all $t \in\left(0, t_{\mathrm{m}}\right)$. Furthermore, we note from (a) that we may infer that if $X_{i: n}$ is new worse than used (NWU) for $i=1,2, \ldots, n$ then $T$ is NWU as well. In a similar manner, we find from (b) that if $X_{i: n}$ is decreasing failure rate (DFR) for $i=1,2, \ldots, n$, then $T$ is DFR as well. For definitions and discussion of the NWU and DFR properties, see [1].

We note that representation (2.1) also holds for systems with exchangeable components (meaning that the distribution of $\left(X_{1}, X_{2}, \ldots, X_{n}\right)$ is permutation invariant) since, in this case, representation (1.1) continues to hold; see [10].

We can obtain another mixture representation using the fact that, in the exchangeable case (and, thus, also in the i.i.d. case), the reliability $\bar{F}_{T}$ of a coherent system $T$ can be written as a generalised mixture (i.e. a mixture which can have some negative coefficients)

$$
\bar{F}_{T}(t)=\sum_{i=1}^{n} a_{i} \bar{F}_{1: i}(t)
$$

where $\bar{F}_{1: i}(t)=\mathrm{P}\left(X_{1: i}>t\right)$ is the reliability function of the series system with $i$ components and the vector of coefficients $\boldsymbol{a}=\left(a_{1}, a_{2}, \ldots, a_{n}\right)$ satisfying $\sum_{i=1}^{n} a_{i}=1$ is referred to as the domination vector or the minimal signature (see [14] and [9], respectively). Then, we obtain

$$
\mathrm{P}(T-t>x \mid T>t)=\sum_{i=1}^{n} a_{i}(t) \bar{F}_{1: i}(x \mid t),
$$


where $\bar{F}_{1: i}(x \mid t)=\mathrm{P}\left(X_{1: i}-t>x \mid X_{1: i}>t\right)$ and $a_{i}(t)=a_{i} \bar{F}_{1: i}(t) / \bar{F}_{T}(t)$. The vector of coefficients $\boldsymbol{a}(t)=\left(a_{1}(t), a_{2}(t), \ldots, a_{n}(t)\right)$ can be called the conditional domination vector, given that $T>t$. Note that some of these coefficients can be negative. For example, the reliability function of $T=\min \left(X_{1}, \max \left(X_{2}, X_{3}\right)\right)$ can be written as

$$
\bar{F}_{T}(t)=2 \bar{F}_{1: 2}(t)-\bar{F}_{1: 3}(t),
$$

that is, its domination vector is $\boldsymbol{a}=(0,2,-1)$; see [9]. Hence, if $T$ has i.i.d. components, the reliability function of its residual lifetime can be written as

$$
\mathrm{P}(T-t>x \mid T>t)=\frac{2}{2-\bar{F}(t)} \bar{F}_{1: 2}(x \mid t)-\frac{\bar{F}(t)}{2-\bar{F}(t)} \bar{F}_{1: 3}(x \mid t),
$$

i.e. its conditional domination vector is

$$
\boldsymbol{a}(t)=\left(0, \frac{2}{2-\bar{F}(t)}, \frac{-\bar{F}(t)}{2-\bar{F}(t)}\right) .
$$

From Table 1 of [9], it is easy to form a table for the conditional domination vectors similar to Table 1 above.

The representation in (2.3) can be used to obtain a third mixture representation based on order statistics obtained from the component residual lifetime distribution as follows.

Theorem 2.5. If $T$ is a coherent system with i.i.d. components then

$$
\mathrm{P}(T-t>x \mid T>t)=\sum_{i=1}^{n} p_{i}^{*}(t) \mathrm{P}\left(Z_{i: n}>x\right),
$$

where $p_{1}^{*}(t), p_{2}^{*}(t), \ldots, p_{n}^{*}(t)$ are coefficients such that $\sum_{i=1}^{n} p_{i}^{*}(t)=1$ and $Z_{i: n}$ is the ith order statistic from i.i.d. random variables with common reliability function $\bar{G}_{t}(x)=\bar{F}(x+$ $t) / \bar{F}(t)$.

The proof is obtained from (2.3) and by using the facts that

$$
\bar{F}_{1: i}(x \mid t)=\mathrm{P}\left(X_{1: i}-t>x \mid X_{1: i}>t\right)=\frac{(\bar{F}(t+x))^{i}}{(\bar{F}(t))^{i}}=\left(\bar{G}_{t}(x)\right)^{i}=\mathrm{P}\left(Z_{1: i}>x\right)
$$

and that $\mathrm{P}\left(Z_{1: i}>x\right)$ can be written as a mixture of $\mathrm{P}\left(Z_{j: n}>x\right)$ for $j=1,2, \ldots, n$ (see [10]). Denote the vector of coefficients in (2.6) by $\boldsymbol{p}^{*}(t)$. Note that the representation in (2.6) also holds for mixed systems and, in particular, for systems with fewer than $n$ components. It may also be noted that Theorem 1.2 can be applied to this representation (when the coefficients are nonnegative). The vectors of coefficients $\boldsymbol{p}^{*}(t)$ in (2.6) with $n=3$ for coherent systems with 1-3 i.i.d. components are presented in Table 2 . Note that $\boldsymbol{p}(t)$ and $\boldsymbol{p}^{*}(t)$ are not necessarily equal (see Tables 1 and 2).

For systems with i.i.d. exponential components, we have the following result.

Corollary 2.1. If $T$ is a coherent system with i.i.d. exponential components then

$$
\mathrm{P}(T-t>x \mid T>t)=\sum_{i=1}^{n} p_{i}^{*}(t) \mathrm{P}\left(X_{i: n}>x\right),
$$

where $p_{1}^{*}(t), p_{2}^{*}(t), \ldots, p_{n}^{*}(t)$ are coefficients such that $\sum_{i=1}^{n} p_{i}^{*}(t)=1$. 
TABLE 2: Vectors of coefficients in (2.6) with $n=3$ for coherent systems with 1-3 i.i.d. components.

\begin{tabular}{ccc}
\hline System & $T=\phi\left(X_{1}, X_{2}, X_{3}\right)$ & $p^{*}(t)$ \\
\hline 1 & $X_{1: 1}=X_{1}$ & $\left(\frac{1}{3}, \frac{1}{3}, \frac{1}{3}\right)$ \\
2 & $X_{1: 2}=\min \left(X_{1}, X_{2}\right)$ & $\left(\frac{2}{3}, \frac{1}{3}, 0\right)$ \\
3 & $X_{2: 2}=\max \left(X_{1}, X_{2}\right)$ & $\left(\frac{2-2 \bar{F}(t)}{6-3 \bar{F}(t)}, \frac{2-\bar{F}(t)}{6-3 \bar{F}(t)}, \frac{2}{6-3 \bar{F}(t)}\right)$ \\
4 & $X_{1: 3}=\min \left(X_{1}, X_{2}, X_{3}\right)$ & $\left(\frac{4-3 \bar{F}(t)}{6-3 \bar{F}(t)}, \frac{2}{6-3 \bar{F}(t)}, 0\right)$ \\
5 & $\min \left(X_{1}, \max \left(X_{2}, X_{3}\right)\right)$ & $\left(\frac{2-2 \bar{F}(t)}{3-2 \bar{F}(t)}, \frac{1}{3-2 \bar{F}(t)}, 0\right)$ \\
6 & $X_{2: 3}(2-$ out-of-3) & $(1,0)$ \\
7 & $\max \left(X_{1}, \min \left(X_{2}, X_{3}\right)\right)$ & $\left(\frac{1+2 \bar{F}(t)-3 \bar{F}^{2}(t)}{3+3 \bar{F}(t)-3 \bar{F}^{2}(t)}, \frac{1+\bar{F}(t)}{3+3 \bar{F}(t)-3 \bar{F}^{2}(t)}, \frac{1}{3+3 \bar{F}(t)-3 \bar{F}^{2}(t)}\right)$ \\
8 & $X_{3: 3}=\max \left(X_{1}, X_{2}, X_{3}\right)$ & $\left(\frac{1-2 \bar{F}(t)+\bar{F}^{2}(t)}{3-3 \bar{F}(t)+\bar{F}^{2}(t)}, \frac{1-\bar{F}(t)}{3-3 \bar{F}(t)+\bar{F}^{2}(t)}, \frac{1}{3-3 \bar{F}(t)+\bar{F}^{2}(t)}\right)$ \\
\hline
\end{tabular}

The proof is immediate since the exponential distribution has the lack of memory property so that $\bar{G}_{t}(x)=\bar{F}(x)$. Observe that this corollary implies that if a system with lifetime $T$ has exponential components, then $(T-t \mid T>t)$ is equal in distribution to the mixture of order statistics with mixing distribution given by $\boldsymbol{p}^{*}(t)$ whenever $p_{i}^{*}(t) \geq 0$ for all $i=1,2, \ldots, n$. The next example shows how this representation can be used to obtain increasing failure rate (IFR) properties and to compare the residual lifetime $(T-t \mid T>t)$ of used systems for different values of $t$ by comparing their vectors of coefficients. It also illustrates how we may compute $\boldsymbol{p}^{*}(t)$.

Example 2.2. Let us consider the system with lifetime $T=\min \left(X_{1}, \max \left(X_{2}, X_{3}\right)\right)$, and let us assume that $X_{1}, X_{2}, X_{3}$ have i.i.d. exponential distributions. The system's signature is $\boldsymbol{s}=\left(\frac{1}{3}, \frac{2}{3}, 0\right)$ and its domination vector is $\boldsymbol{a}=(0,2,-1)$, that is, its reliability function $\bar{F}_{T}(t)=\mathrm{P}(T>t)$ can be written as (2.4) and the reliability of its residual lifetime as (2.5). If the components have i.i.d. exponential distributions then $\bar{F}_{1: i}(x \mid t)=\mathrm{P}\left(X_{1: i}>x\right)$. Moreover, since the signatures of order 3 of $X_{1: 2}$ and $X_{1: 3}$ are $s_{1: 2}=\left(\frac{2}{3}, \frac{1}{3}, 0\right)$ and $s_{1: 3}=(1,0,0)$, respectively (see [10]), we have

$$
\mathrm{P}(T-t>x \mid T>t)=\frac{4-3 \bar{F}(t)}{6-3 \bar{F}(t)} \bar{F}_{1: 3}(x)+\frac{2}{6-3 \bar{F}(t)} \bar{F}_{2: 3}(x),
$$

that is,

$$
p^{*}(t)=\left(\frac{4-3 \bar{F}(t)}{6-3 \bar{F}(t)}, \frac{2}{6-3 \bar{F}(t)}, 0\right) .
$$

Note that $\boldsymbol{p}^{*}(0)=\boldsymbol{s}=\left(\frac{1}{3}, \frac{2}{3}, 0\right)$ and that $\lim _{t \rightarrow \infty} \boldsymbol{p}^{*}(t)=\boldsymbol{s}_{1: 2}=\left(\frac{2}{3}, \frac{1}{3}, 0\right)$ (i.e. it is asymptotically equivalent to a two-component series system). Moreover, since

$$
\boldsymbol{s} \geq_{\text {st }} \boldsymbol{p}^{*}\left(t_{1}\right) \geq_{\text {st }} \boldsymbol{p}^{*}\left(t_{2}\right) \geq_{\text {st }} \boldsymbol{s}_{1: 2} \quad \text { for all } 0 \leq t_{1} \leq t_{2},
$$


then from Theorem 1.2 we have

$$
T \geq_{\text {st }}\left(T-t_{1} \mid T>t_{1}\right) \geq_{\text {st }}\left(T-t_{2} \mid T>t_{2}\right) \geq_{\text {st }} X_{1: 2} \quad \text { for all } 0 \leq t_{1} \leq t_{2} .
$$

Therefore, $T$ is new better than used (NBU). It is a well-known property that the coherent systems with NBU components are NBU. Actually, the used systems above are st-ordered in $t$ (i.e. the older the system is, the worse off it is). This implies that $T$ is IFR; see [15, p. 15]. However, if we consider its dual system $T^{*}=\max \left(X_{1}, \min \left(X_{2}, X_{3}\right)\right.$ ) (system 7 in Table 2) with i.i.d. exponential components then it is easy to show that $s \geq_{\mathrm{st}} \boldsymbol{p}^{*}(t)$ for all $t$ (i.e. $T^{*}$ is NBU), that $\boldsymbol{p}^{*}\left(t_{1}\right) \geq_{\text {st }} \boldsymbol{p}^{*}\left(t_{2}\right)$ for all $0 \leq t_{1} \leq t_{2} \leq t_{0}$, where $\bar{F}\left(t_{0}\right)=0.5$, and that $\boldsymbol{p}^{*}(t) \leq_{\text {st }} \boldsymbol{p}^{*}\left(t^{\prime}\right)$ for all $t_{3} \leq t_{1} \leq t_{2}$, where $\bar{F}\left(t_{3}\right)=-2+\sqrt{5}$. Therefore, it is not IFR. Actually, the system improves when $t$ increases for $t \geq t_{3}$. This example shows that Lemma 2.1 does not hold for $\boldsymbol{p}^{*}(t)$.

The next example shows that the representation in (2.7) need not hold when the components have nonexponential distributions.

Example 2.3. Let us consider again the system $T=\min \left(X_{1}, \max \left(X_{2}, X_{3}\right)\right)$, and let us now assume that it has i.i.d. components with common reliability function $\bar{F}(t)=1 /(1+t)$ for $t \geq 0$. Then,

$$
\bar{F}_{T}(t)=2 \bar{F}^{2}(t)-\bar{F}^{3}(t)=\frac{2}{(1+t)^{2}}-\frac{1}{(1+t)^{3}}
$$

and

$$
\mathrm{P}(T-t>x \mid T>t)=\frac{1+2 t+2 x}{1+2 t} \frac{(1+t)^{3}}{(1+t+x)^{3}} .
$$

From [10], $\mathrm{P}(T-t>x \mid T>t)$ is a generalised mixture of $\bar{F}_{1: 3}, \bar{F}_{2: 3}, \bar{F}_{3: 3}$ if and only if the system above is a generalised mixture of $\bar{F}_{1: 1}, \bar{F}_{1: 2}, \bar{F}_{1: 3}$. Let us suppose that it is a generalised mixture of $\bar{F}_{1: 1}, \bar{F}_{1: 2}, \bar{F}_{1: 3}$, that is,

$$
\mathrm{P}(T-t>x \mid T>t)=c_{1}(t) \frac{1}{1+x}+c_{2}(t) \frac{1}{(1+x)^{2}}+c_{3}(t) \frac{1}{(1+x)^{3}} \quad \text { for all } x \geq 0,
$$

for some coefficients $c_{1}(t), c_{2}(t)$, and $c_{3}(t)$ such that $c_{1}(t)+c_{2}(t)+c_{3}(t)=1$. If $y=1+x$, this last expression is equivalent to

$$
(2 t+2 y-1) y^{3}=\left(d_{1}(t) y^{2}+d_{2}(t) y+d_{3}(t)\right)(t+y)^{3} \quad \text { for all } y \geq 1,
$$

where $d_{i}(t)$ depends only on $t$ for $i=1,2,3$. It is easy to see that this expression cannot be true for all $y \geq 1$.

\section{Mixture representations of residual lifetimes of used systems with additional information}

Let us now assume that the system is working at time $t$, i.e. $\{T>t\}$, and that we have some additional information at that time point. For example, a logical condition that could arise in practical situations is that, at age $t$, we know that the system is working and that at least $i$ of the components have failed, i.e. $\left\{X_{i: n}<t\right\}$. The results included in this section extend some of the results given in [4] to the present scenario. Thus, we have

$$
\mathrm{P}\left(T-t>x \mid T>t, X_{i: n}<t\right)=\frac{\mathrm{P}\left(T>t+x, X_{i: n}<t\right)}{\mathrm{P}\left(T>t, X_{i: n}<t\right)}
$$


for all $x \geq 0$ and $t \geq 0$ such that $\mathrm{P}\left(T>t, X_{i: n}<t\right)>0$. To obtain a mixture representation similar to the one in (2.1) for this case, we need the following lemma.

Lemma 3.1. If $T$ is the lifetime of a coherent system and $i(1 \leq i<n)$ is an integer, then

$$
\mathrm{P}\left(X_{i: n}<x, T>y\right)=\sum_{k=1}^{n-i} \sum_{j=i}^{n-k} a_{j, k} F^{j}(x) \bar{F}^{k}(y) \quad \text { for all } x \leq y,
$$

where the coefficients $a_{j, k}$ are real numbers that do not depend on $F$.

Proof. It is well known that the lifetime $T$ of a coherent system can be written as $T=$ $\max _{j=1,2, \ldots, s} \min _{i \in P_{j}} X_{i}$, where $P_{1}, P_{2}, \ldots, P_{s}$ are the minimal path sets of the system; see, for example, [1, p. 12]. We shall use the following notation in our proof: $X_{P}=\min _{i \in P} X_{i}$ and $X^{Q}=\max _{i \in Q} X_{i}$. It follows from the inclusion-exclusion formula that

$$
\begin{aligned}
\mathrm{P}\left(X_{i: n}<x, T>y\right)= & \mathrm{P}\left(\left\{X_{i: n}<x\right\} \cap\left(\bigcup_{j=1}^{s}\left\{X_{P_{j}}>y\right\}\right)\right) \\
= & \mathrm{P}\left(\bigcup_{j=1}^{s}\left(\left\{X_{i: n}<x\right\} \cap\left\{X_{P_{j}}>y\right\}\right)\right) \\
= & \sum_{j=1}^{s} \mathrm{P}\left(X_{i: n}<x, X_{P_{j}}>y\right)-\sum_{j<k} \mathrm{P}\left(X_{i: n}<x, X_{P_{j} \cup P_{k}}>y\right) \\
& +\cdots \pm \mathrm{P}\left(X_{i: n}<x, X_{P_{1} \cup P_{2} \cup \cdots \cup P_{s}}>y\right) .
\end{aligned}
$$

Analogously, $X_{i: n}=\min _{j=1,2, \ldots, m} \max _{k \in Q_{j}} X_{k}$, where $m=\left(\begin{array}{c}n \\ i\end{array}\right)$ and $Q_{1}, Q_{2}, \ldots, Q_{m}$ are all the subsets of $\{1,2, \ldots, n\}$ with exactly $i$ elements. Therefore, if $I \subseteq\{1,2, \ldots, n\}$ such that $|I| \leq n-i$, where $|I|$ denotes the cardinality of $I$, then

$$
\begin{aligned}
& \mathrm{P}\left(X_{i: n}<x, X_{I}>y\right)=\mathrm{P}\left(\left\{X_{I}>y\right\} \cap\left(\bigcup_{j=1}^{m}\left\{X^{Q_{j}}<x\right\}\right)\right) \\
& =\mathrm{P}\left(\bigcup_{j=1}^{m}\left(\left\{X^{Q_{j}}<x\right\} \cap\left\{X_{I}>y\right\}\right)\right) \\
& =\sum_{j=1}^{m} \mathrm{P}\left(X^{Q_{j}}<x, X_{I}>y\right)-\sum_{j<k} \mathrm{P}\left(X^{Q_{j} \cup Q_{k}}<x, X_{I}>y\right) \\
& +\cdots \pm \mathrm{P}\left(X^{Q_{1} \cup Q_{2} \cup \cdots \cup Q_{m}}<x, X_{I}>y\right) \\
& =\sum_{j=1}^{m^{*}} \mathrm{P}\left(X^{Q_{j}^{*}}<x, X_{I}>y\right)-\sum_{j<k} \mathrm{P}\left(X^{Q_{j}^{*} \cup Q_{k}^{*}}<x, X_{I}>y\right) \\
& +\cdots \pm \mathrm{P}\left(X^{Q_{1}^{*} \cup Q_{2}^{*} \cup \cdots \cup Q_{m^{*}}^{*}}<x, X_{I}>y\right),
\end{aligned}
$$

where $m^{*}=\left(\begin{array}{c}|\bar{I}| \\ i\end{array}\right)$ and $Q_{1}^{*}, Q_{2}^{*}, \ldots, Q_{m^{*}}^{*}$ are all the subsets of $\bar{I}=\{1,2, \ldots, n\}-I$ with exactly $i$ elements. Now, upon using the fact that the components are i.i.d., from (3.4) we obtain

$$
\mathrm{P}\left(X_{i: n}<x, X_{I}>y\right)=c_{i} F^{i}(x) \bar{F}^{|I|}(y)+c_{i+1} F^{i+1}(x) \bar{F}^{|I|}(y)+\cdots+c_{|\bar{I}|} F^{|\bar{I}|}(x) \bar{F}^{|I|}(y),
$$


where $c_{i}, c_{i+1}, \ldots, c_{|\bar{I}|}$ are coefficients that do not depend on $F$. Using this last expression and (3.3), we obtain (3.2) upon grouping together terms with the same powers of $F$ and $\bar{F}$ (note that $\mathrm{P}\left(X_{i: n}<x, X_{I}>y\right)=0$ whenever $\left.|I|>n-i\right)$.

Since mixed systems are stochastic mixtures of coherent systems, Lemma 3.1 also holds for mixed systems. We now establish the main theorem of this section which shows that the reliability function of $\left(T-t \mid T>t, X_{i: n}<t\right)$ admits a representation similar to the one in $(2.1)$ for $i=1,2, \ldots, n-1$ such that $\mathrm{P}\left(T>t, X_{i: n}<t\right)>0$.

Theorem 3.1. If $T$ is the lifetime of a mixed system with i.i.d. components having a common continuous distribution function $F$ and $i \in\{1,2, \ldots, n-1\}$ such that $\mathrm{P}\left(T>t, X_{i: n}<\right.$ $t)>0$, then there exist coefficients $p_{1}(t, i), p_{2}(t, i), \ldots, p_{n}(t, i)$ (that depend on $F$ ) such that $\sum_{j=1}^{n} p_{j}(t, i)=1$ and

$$
\mathrm{P}\left(T-t>x \mid T>t, X_{i: n}<t\right)=\sum_{j=1}^{n} p_{j}(t, i) \mathrm{P}\left(X_{j: n}-t>x \mid X_{j: n}>t\right)
$$

for all $x \geq 0$.

Proof. From (3.1) and Lemma 3.1, we have

$$
\mathrm{P}\left(T-t>x \mid T>t, X_{i: n}<t\right)=\frac{\sum_{k=1}^{n-i} \sum_{j=i}^{n-k} a_{j, k} F^{j}(t) \bar{F}^{k}(x+t)}{\sum_{k=1}^{n-i} \sum_{j=i}^{n-k} a_{j, k} F^{j}(t) \bar{F}^{k}(t)},
$$

where the coefficients $a_{j, k}$ do not depend on $F$. Therefore,

$$
\mathrm{P}\left(T-t>x \mid T>t, X_{i: n}<t\right)=\sum_{k=1}^{n-i} \sum_{j=i}^{n-k} a_{j, k}(t, i) \mathrm{P}\left(X_{1: k}-t>x \mid X_{1: k}>t\right),
$$

where $\mathrm{P}\left(X_{1: k}-t>x \mid X_{1: k}>t\right)=\bar{F}^{k}(x+t) / \bar{F}^{k}(t)$ and

$$
a_{j, k}(t, i)=\frac{a_{j, k} F^{j}(t) \bar{F}^{k}(t)}{\sum_{k=1}^{n-i} \sum_{j=i}^{n-k} a_{j, k} F^{j}(t) \bar{F}^{k}(t)} .
$$

Hence, if $a_{k}(t, i)=\sum_{j=i}^{n-k} a_{j, k}(t, i)$, from (3.6) we obtain

$$
\mathrm{P}\left(T-t>x \mid T>t, X_{i: n}<t\right)=\sum_{k=1}^{n-i} a_{k}(t, i) \mathrm{P}\left(X_{1: k}-t>x \mid X_{1: k}>t\right) .
$$

Finally, since, from (2.1), $\mathrm{P}\left(X_{1: k}-t>x \mid X_{1: k}>t\right)$ can be written as a mixture of $\mathrm{P}\left(X_{i: n}-t>\right.$ $\left.x \mid X_{i: n}>t\right)$ for $i=1,2, \ldots, n$, we obtain the required result.

The vector of coefficients $\boldsymbol{p}(t, i)=\left(p_{1}(t, i), p_{2}(t, i), \ldots, p_{n}(t, i)\right)$ in (3.5) can therefore be called the conditional domination under the condition $\left\{X_{i: n}<t<T\right\}$. Note that the coefficients $p_{j}(t, i)$ need not all be nonnegative. If they are all nonnegative then Theorem 2.1 can also be applied to this new mixture representation. Also, note that we have a similar mixture representation (given in (3.7)) in terms of the reliability functions of the residual lifetimes of series systems. The vector of coefficients in that representation can be represented by $\boldsymbol{a}(t, i)$. The following examples show how these coefficients can be computed in specific cases. They also show that some coefficients can be negative. 
Example 3.1. Let us consider again the system $T=\min \left(X_{1}, \max \left(X_{2}, X_{3}\right)\right)$, and let us assume that, at age $t$, the system is working and that at least one component has failed, i.e. $\{T>$ $\left.t, X_{1: 3}<t\right\}$. The minimal path sets of $T$ are $P_{1}=\{1,2\}$ and $P_{2}=\{1,3\}$. Then, for $x \geq 0$, we have

$$
\begin{aligned}
\mathrm{P}\left(X_{1: 3}<t, T>t+x\right)= & \mathrm{P}\left(X_{1: 3}<t, X_{\{1,2\}}>x+t\right)+\mathrm{P}\left(X_{1: 3}<t, X_{\{1,3\}}>x+t\right) \\
& -\mathrm{P}\left(X_{1: 3}<t, X_{1: 3}>x+t\right) \\
= & 2 F(t) \bar{F}^{2}(x+t) .
\end{aligned}
$$

Therefore,

$$
\mathrm{P}\left(T-t>x \mid T>t, X_{1: 3}<t\right)=\frac{\bar{F}^{2}(x+t)}{\bar{F}^{2}(t)}=\mathrm{P}\left(X_{1: 2}-t>x \mid X_{1: 2}>t\right),
$$

that is, $\boldsymbol{a}(t, 1)=(0,1,0)$. Hence, the system is equal in law to the residual life of a twocomponent series system (an intuitive property). Finally, as the vector $\boldsymbol{p}_{1: 2}(t)$ of coefficients in (2.1) of $X_{1: 2}$ with $n=3$ is $\boldsymbol{p}_{1: 2}(t)=\left(\frac{2}{3} \bar{F}(t), 1-\frac{2}{3} \bar{F}(t), 0\right)$ (see Table 1), we have

$$
\mathrm{P}\left(T-t>x \mid T>t, X_{1: 3}<t\right)=\frac{2 \bar{F}(t)}{3} \bar{F}_{1: 3}(x \mid t)+\frac{3-2 \bar{F}(t)}{3} \bar{F}_{2: 3}(x \mid t),
$$

where $\bar{F}_{i: 3}(x \mid t)=\mathrm{P}\left(X_{i: 3}-t>x \mid X_{i: 3}>t\right)$, that is,

$$
\boldsymbol{p}(t, 1)=\left(\frac{2 \bar{F}(t)}{3}, \frac{3-2 \bar{F}(t)}{3}, 0\right) .
$$

Naturally, we can use this representation to study what the effect of the condition $\left\{X_{1: 3}<t\right\}$ is on the used system $(T-t \mid T>t)$. Since the vector of coefficients of $(T-t \mid T>t)$ in $(2.1)$ is

$$
\boldsymbol{p}(t)=\left(\frac{\bar{F}(t)}{6-3 \bar{F}(t)}, \frac{6-4 \bar{F}(t)}{6-3 \bar{F}(t)}, 0\right)
$$

(see Table 1) and $\boldsymbol{p}(t, 1) \leq_{\text {st }} \boldsymbol{p}(t)$, from Theorem 2.1 we have

$$
\left(T-t \mid T>t, X_{1: 3}<t\right) \leq_{\text {st }}(T-t \mid T>t) \text { for all } t \geq 0
$$

(i.e. as we would expect, $\left\{X_{1: 3}<t\right\}$ adversely affects the used system).

Example 3.2. Let us consider the parallel system with lifetime $X_{3: 3}$, and suppose that, at age $t$, the system is working and that at least one component has failed, i.e. $\left\{X_{1: 3}<t<X_{3: 3}\right\}$. Then, we have

$$
\mathrm{P}\left(X_{1: 3}<t, X_{3: 3}>x+t\right)=6 F(t) \bar{F}(x+t)-3 F^{2}(t) \bar{F}(x+t)-3 F(t) \bar{F}^{2}(x+t)
$$

and

$$
\mathrm{P}\left(X_{3: 3}-t>x \mid X_{1: 3}<t, X_{3: 3}>t\right)=(1+\bar{F}(t)) \bar{F}_{1: 1}(x \mid t)-\bar{F}(t) \bar{F}_{1: 2}(x \mid t),
$$

where $\bar{F}_{1: i}(x \mid t)=\mathrm{P}\left(X_{1: i}-t>x \mid X_{1: i}>t\right)$ for $i=1,2$. The vectors of coefficients in (2.1) with $n=3$ of $X_{1: 1}$ and $X_{1: 2}$ are

$$
p_{1: 1}(t)=\left(\frac{1}{3} \bar{F}^{2}(t), \bar{F}(t)-\frac{2}{3} \bar{F}^{2}(t), 1-\bar{F}(t)+\frac{1}{3} \bar{F}^{2}(t)\right)
$$


and

$$
p_{1: 2}(t)=\left(\frac{2}{3} \bar{F}(t), 1-\frac{2}{3} \bar{F}(t), 0\right),
$$

respectively (see Table 1$)$. Therefore, the vector $\boldsymbol{p}_{3: 3}(t, 1)$ of coefficients of $\left(X_{3: 3}-t \mid X_{1: 3}<\right.$ $t, X_{3: 3}>t$ ) in (3.5) with $n=3$ and $i=1$ is given by

$$
p_{3: 3}(t, 1)=\left(\frac{1}{3} \bar{F}^{3}(t)-\frac{1}{3} \bar{F}^{2}(t), \bar{F}^{2}(t)-\frac{2}{3} \bar{F}^{3}(t), 1-\frac{2}{3} \bar{F}^{2}(t)+\frac{1}{3} \bar{F}^{3}(t)\right) .
$$

Observe that the first coefficient is negative. Also, note that $\left(X_{3: 3}-t \mid X_{1: 3}<t, X_{3: 3}>t\right)$ is not equal in distribution to $\left(X_{2: 2}-t \mid X_{2: 2}>t\right)$. However, a straightforward calculation shows that $\left(X_{3: 3}-t \mid X_{1: 3}<t, X_{3: 3}>t\right) \leq_{\text {st }}\left(X_{3: 3}-t \mid X_{3: 3}>t\right)$.

Note that Theorem 2.1 cannot be applied to representations with some negative coefficients. To extend this theorem, we therefore need the following result on generalised mixtures. For this purpose, we need the following partial order in $\mathbb{R}^{n}$. If $\boldsymbol{x}=\left(x_{1}, x_{2}, \ldots, x_{n}\right)$ and $\boldsymbol{y}=$ $\left(y_{1}, y_{2}, \ldots, y_{n}\right)$ are two vectors of real numbers then $\boldsymbol{x}=\left(x_{1}, x_{2}, \ldots, x_{n}\right)$ is greater than or equal to $\boldsymbol{y}=\left(y_{1}, y_{2}, \ldots, y_{n}\right)$ in the tail domination order (written as $\boldsymbol{x} \geq_{\operatorname{td}} \boldsymbol{y}$ ) if $\sum_{i=1}^{j} x_{i} \leq$ $\sum_{i=1}^{j} y_{i}$ for $j=1,2, \ldots, n-1$ and $\sum_{i=1}^{n} x_{i}=\sum_{i=1}^{n} y_{i}$. Note that if $x_{i}, y_{i} \geq 0$ for $i=$ $1,2, \ldots, n$ and $\sum_{i=1}^{n} x_{i}=\sum_{i=1}^{n} y_{i}=1$, then $\boldsymbol{x} \geq_{\mathrm{td}} \boldsymbol{y}$ if and only if $\boldsymbol{x} \geq_{\mathrm{st}} \boldsymbol{y}$.

Theorem 3.2. Let $\bar{F}$ and $\bar{G}$ be two reliability functions such that $\bar{F}=\sum_{i=1}^{n} x_{i} \bar{F}_{i}$ and $\bar{G}=$ $\sum_{i=1}^{n} y_{i} \bar{F}_{i}$, where $\bar{F}_{1}, \bar{F}_{2}, \ldots, \bar{F}_{n}$ are reliability functions and $x_{i}$ and $y_{i}$ are real numbers satisfying $\sum_{i=1}^{n} x_{i}=\sum_{i=1}^{n} y_{i}=1$. If

$$
\boldsymbol{x}=\left(x_{1}, x_{2}, \ldots, x_{n}\right) \geq_{\mathrm{td}} \boldsymbol{y}=\left(y_{1}, y_{2}, \ldots, y_{n}\right)
$$

and

then $\bar{F} \geq_{\text {st }} \bar{G}$

$$
\bar{F}_{1} \leq_{\text {st }} \bar{F}_{2} \leq_{\text {st }} \cdots \leq_{\text {st }} \bar{F}_{n}
$$

Proof. We need to prove that $\bar{F}(t)=\sum_{i=1}^{n} x_{i} \bar{F}_{i}(t) \geq \bar{G}(t)=\sum_{i=1}^{n} y_{i} \bar{F}_{i}(t)$, which is equivalent to proving that

$$
\sum_{x_{i}>0} x_{i} \bar{F}_{i}(t)-\sum_{y_{i}<0} y_{i} \bar{F}_{i}(t) \geq \sum_{y_{i}>0} y_{i} \bar{F}_{i}(t)-\sum_{x_{i}<0} x_{i} \bar{F}_{i}(t) .
$$

The last expression can be rewritten as $\sum_{i=1}^{n} a_{i} \bar{F}_{i}(t) \geq \sum_{i=1}^{n} b_{i} \bar{F}_{j}(t)$, where $a_{i}=0, x_{i},-y_{i}$, or $x_{i}-y_{i}$ and $b_{i}=0, y_{i},-x_{i}$, or $y_{i}-x_{i}$. Note that $a_{i} \geq 0$ and $b_{i} \geq 0$ for $i=1,2, \ldots, n$. Moreover, as $\boldsymbol{x} \geq_{\mathrm{td}} \boldsymbol{y}, \sum_{i=1}^{j} x_{i} \leq \sum_{i=1}^{j} y_{i}$. Then

$$
\sum_{i=1,2, \ldots, j, x_{i}>0} x_{i}-\sum_{i=1,2, \ldots, j, y_{i}<0} y_{i} \leq \sum_{i=1,2, \ldots, j, y_{i}>0} y_{i}-\sum_{i=1,2, \ldots, j, x_{i}<0} x_{i},
$$

and, hence, $\sum_{i=1}^{j} a_{i} \leq \sum_{i=1}^{j} b_{i}$ for $j=1,2, \ldots, n-1$. Analogously, as $\sum_{i=1}^{n} x_{i}=\sum_{i=1}^{n} y_{i}$, $\sum_{i=1}^{n} a_{i}=\sum_{i=1}^{n} b_{i}=c>0$. Now, if we define $p_{i}=a_{i} / c, q_{i}=b_{i} / c, \boldsymbol{p}=\left(p_{1}, p_{2}, \ldots, p_{n}\right)$, and $\boldsymbol{q}=\left(q_{1}, q_{2}, \ldots, q_{n}\right)$, then $\boldsymbol{p} \geq_{\text {st }} \boldsymbol{q}$. Then, from Theorem 1.A.6 of [15] and $\bar{F}_{1} \leq_{\text {st }} \bar{F}_{2} \leq_{\text {st }}$ $\cdots \leq_{\text {st }} \bar{F}_{n}$, we have

$$
\sum_{i=1}^{n} p_{i} \bar{F}_{i}(t) \geq \sum_{i=1}^{n} q_{i} \bar{F}_{i}(t)
$$

and, hence, $\bar{F} \geq_{\text {st }} \bar{G}$. If the direction of ordering in Theorem 3.2 is reversed (i.e. if $\bar{F}_{1} \geq_{\text {st }}$ $\bar{F}_{2} \geq_{\text {st }} \cdots \geq_{\text {st }} \overline{\bar{F}}_{n}$ ), then we may show that $\bar{F} \leq_{\text {st }} \bar{G}$ by a similar argument. 
Therefore, Theorem 2.1(a) can also now be applied to the mixture representations with negative coefficients obtained from (3.5). For example, from Table 1 and Example 3.2, we have $p_{3: 3}(t, 1) \geq_{\text {st }} p_{2: 2}(t)$ and, hence, $\left(X_{3: 3}-t \mid X_{1: 3}<t, X_{3: 3}>t\right) \geq_{\text {st }}\left(X_{2: 2}-t \mid X_{2: 2}>t\right)$ for all $t \geq 0$.

\section{Conclusions}

The goal of this paper has been to establish representation theorems for the conditional reliability of the residual lifetime of a used system given particular information on the state of the system upon inspection at time $t$. Our results have been obtained under the tacit assumption of i.i.d. component lifetimes of the systems under study. Two natural forms of conditioning are considered, namely, that the system is working at time $t$ (i.e. $T>t$ ) or that the system is working and at least $i$ components have failed by time $t$ (i.e. $\left\{X_{i: n}<t<T\right\}$ ). The expressions in (2.1) and (3.5) constitute such representations in terms of the corresponding conditional reliability of the residual lifetimes of the order statistics of the system's components. The utility of these representations is illustrated in several examples in which the systems' conditional residual reliabilities are computed and compared. Alternative representation results in terms of the ordered remaining life of surviving components are also explored. Possible extensions to systems whose component lifetimes are exchangeable are duly noted. Finally, several new preservation theorems are established, thereby providing sufficient conditions for various forms of stochastic relations between the residual lifetimes of two systems of interest. In ongoing studies we are examining two interesting extensions of the work above. One investigation is centred upon alternative formulations of the information available at inspection. We are also exploring the possibility of obtaining useful representation results in the challenging problem in which systems have components whose lifetimes are independent but not necessarily identically distributed. Finally, what can be said about the general case of dependent components remains an interesting open question.

\section{Acknowledgements}

The authors are very grateful to the anonymous referee for several helpful comments. JN, NB, and FJS acknowledge financial support for this research from the Ministerio de Ciencia y Tecnología (under grant MTM2006-12834), the Natural Sciences and Engineering Research Council of Canada, and the US Army Research Office (under grants WN11NF-05-1-0118 and WN11NF-08-0077), respectively.

\section{References}

[1] Barlow, R. E. and Proschan, F. (1975). Statistical Theory of Reliability and Life Testing. Holt, Rinehart and Winston, New York.

[2] Boland, P. J. And Samaniego, F. (2004). The signature of a coherent system and its applications in reliability. In Mathematical Reliability: An Expository Perspective, eds R. Soyer, T. Mazzuchi and N. D. Singpurwalla, Kluwer, Boston, pp. 1-29.

[3] David, H. A. and Nagaraja, H. N. (2003). Order Statistics, 3rd edn. Wiley, Hoboken, New Jersey.

[4] Khaledi, B. E. and Shaked, M. (2007). Ordering conditional lifetimes of coherent systems. J. Statist. Planning Infer. 137, 1173-1184.

[5] Kochar, S., Mukerjee, H., and Samaniego, F. J. (1999). The "signature" of a coherent system and its application to comparison among systems. Naval Res. Logistics 46, 507-523.

[6] Navarro, J. (2008). Likelihood ratio ordering of order statistics, mixtures and systems. J. Statist. Planning Infer. 138, 1242-1257.

[7] Navarro, J. and Eryilmaz, S. (2007). Mean residual lifetimes of consecutive- $k$-out-of- $n$ systems. J. Appl. Prob. 44, 82-98. 
[8] Navarro, J. and Hernandez, P. J. (2008). Mean residual life functions of finite mixtures, order statistics and coherent systems. Metrika 67, 277-298.

[9] Navarro, J., Ruiz, J. M. and Sandoval, C.J. (2007). Properties of coherent systems with dependent components. Commun. Statist. Theory Meth. 36, 175-191.

[10] Navarro, J., Samaniego, F. J., Balakrishnan, N. and Bhattacharya, D. (2008). On the application and extension of system signatures to problems in engineering reliability. Naval Res. Logistics 55, 313-327.

[11] Navarro, J. And Shaked, M. (2006). Hazard rate ordering of order statistics and systems. J. Appl. Prob. 43, 391-408.

[12] Samaniego, F. J. (1985). On closure of the IFR class under formation of coherent systems. IEEE Trans. Reliab. 34, 69-72.

[13] Samaniego, F. (2007). System Signatures and Their Applications in Engineering Reliability (Internat. Ser. Operat. Res. Manag. Sci. 110). Springer, New York.

[14] Satyarananaya, A. and Prabhakar, A. (1978). A new topological formula and rapid algorithm for reliability analysis of complex networks. IEEE Trans. Reliab. 30, 82-100.

[15] Shaked, M. and Shanthikumar, J. G. (2007). Stochastic Orders. Springer, New York. 